\title{
Screening of Fungal Endopohytes against Soil-borne Fungal Pathogens in Tomato
}

\author{
Basamma Hadimani* and S.T. Naik \\ Department of Plant Pathology, University of Agricultural Sciences, \\ Dharwad- 580 005, Karnataka, India \\ *Corresponding author
}

\section{A B S T R A C T}

Keywords

Fungal endophytes, Sclerotium rolfsii, Rhizoctonia solani, Fusarium solani and dual culture method

Article Info

Accepted:

26 June 2018

Available Online:

10 July 2018

\begin{abstract}
A total of 66 fungal endophytes were isolated from apparently healthy tomato plant parts viz., root, stem and leaf tissues and evaluated against soil- borne pathogens viz., Sclerotium rolfsii, Rhizoctonia solani and Fusarium solani by dual culture method to test antifungal activity of tomato fungal endophytes. In dual culture method, among the 66 fungal isolates, irrespective of isolates from root, stem and leaf, some (RFHHO-7, RFHKM-9, RFDHE-10, RFBBA-23, SFDOF-11, SFDDE-12, LFDHO-3 and LFDLA-9) have shown maximum mycelial inhibition of three pathogens. Isolates RFBBE-19 and RFDUN-22 were fast growing isolates and were more effective against $F$. solani and $R$. solani as compared to $S$. rolfsii. Isolate LFDKA-20 though showed the minimum inhibition against all pathogens as compared to other effective isolates but it also showed the clear inhibition zone which may be due to the production of antimicrobial compounds from the endophyte. From the results of present study it is concluded that the possible role of growth inhibition by the endophytes was attributed to the production of diffusible and volatile metabolites, lytic enzymes and by competition with pathogens.
\end{abstract}

\section{Introduction}

Among the vegetables tomato is the second most consumed and widely grown vegetable in the world after potato. Tomato is popular fresh and in many processed forms (e.g., ketchup, canned whole or in pieces, puree, sauce, soup and juice). The ripe fruits are good source of vitamin $\mathrm{A}, \mathrm{B}$ and $\mathrm{C}$ which add wide varieties of colour and flavour to the food (Dias, 2012). At present, the total tomato production in India is about 19.70 million tonnes from 0.808 million ha area with productivity of 24.4 tonnes per hectare. In Karnataka, tomato occupies 63.73 thousand ha with a production of 2138.13 thousand metric tonnes having productivity of 33.55 tonnes per hectare (Anon., 2017). Among the pathogens that affect the tomato crop, soil-borne fungal pathogens, including species belonged to Sclerotium, Fusarium, Pythium, Rhizoctonia, and Verticillium genera causing the root rot or damping- off and wilt which affect the quality with yield reduction. $S$. rolfsii reported yield 
loss up to 30 per cent (Mandal et al., 2017), $R$. solani causes up to 30 per cent (Muriungi et al., 2014) and F. oxysporum f.sp. lycopersici causes 10 -90 per cent in tomato (Kumar and Sharma, 2015). Some of these pathogens are particularly challenging because they often survive in soil for many years (Steven, 2003). To manage such diseases, farmers presently use different fungicides formulations at least for 8-10 times in one growing season which has resulted in several undesirable effects like pesticide pollution, fungicide resistance, elimination of beneficial fauna, environmental pollution and human health hazards (Kumar and Sharma, 2015). So integrated disease management where biological control is one practice is becoming key consideration for soil- borne diseases. Use of endophytes as biocontrol agent may open up new area of research in plant protection in the recent decades under various agro-climatic situations. Endophytes are plant associated microorganisms that live inside plant tissues without causing any harm to plants. The interest in endophytic research has increased, as they colonize the internal tissues of their host plants and improve plant tolerance to various abiotic stress factors and can protect plants from various pathogenic microbes (Pawle and Singh, 2014). With this view in present study an attempt was made to isolate fungal endophytes and evaluated them under in vitro condition by dual culture. A total of 66 fungal endophytes were isolated and evaluated against $S$. rolfsii, $R$. solani and $F$. solani by dual culture method to test antifungal activity of fungal endophytes.

\section{Materials and Methods}

\section{Isolation of bacterial endophytes}

A survey was conducted during 2016-2017 to isolate fungal endophytes in tomato. Apparently healthy leaves, stems and root samples from tomato crop were collected from fields in Belagavi, Dharwad and Haveri districts of northern Karnataka. Roots, stems and leaf samples collected were washed in running tap water to remove soil dirt and debris and cut into $1 \mathrm{~cm}$ sections. After this, surface sterilization was done with 70 per cent ethanol for a minute followed by 1 per cent sodium hypochlorite for 3 minutes. Subsequently the sections were rinsed with sterile distilled water and placed on $9 \mathrm{~cm}$ Petri plates containing potato dextrose agar (PDA) medium amended with streptomycin (250 $\mathrm{mg} / \mathrm{l}$ ) to slow down the bacterial growth. Sterilized tissue segments were pressed onto the surface of PDA medium to check the efficacy of surface sterilization procedure and to confirm endophytic isolations only from internal tissues of the plant segments. The absence of growth of any fungi on the medium confirmed that the surface sterilization procedure was effective in removing the surface fungi (Schulz et al., 1993). All plates were incubated at $25 \pm 1{ }^{\circ} \mathrm{C}$ and observed for fungal growth at 3 days interval for a duration upto 7 - 10 days. Fungi growing out from the plant tissues were transferred on to fresh PDA medium. After purifying the isolates for several times, final pure cultures were transferred on to PDA slants and stored in refrigerator at $4^{\circ} \mathrm{C}$ for further studies.

\section{Antifungal activity of fungal endophytes by dual culture metho}

Dual culture techniqueeas adopted for antagonistic activity of isolated endophytes against $S$. rolfsii, $R$. solani and $F$. solani on PDA plates (Deepa and Sally, 2015). In dual culture technique twenty $\mathrm{ml}$ of sterilized and cooled PDA was poured into sterilized Petri plates. Fungal endophytes were evaluated by inoculating the pathogen at one side of Petri plate and the fungal endophyte inoculated at exactly opposite side of the same plate by leaving 3-4 cm gap. For this, actively growing cultures were used with three replications. 
After required period of incubation i.e., after growth of colony in control plate reached 90 $\mathrm{mm}$ diameter, the radial growth of pathogen in treated plate was measured. Per cent inhibition over control was worked out according to formula given by Vincent (1947).

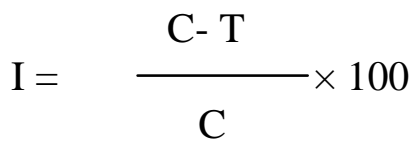

Where, $\mathrm{I}=$ Per cent inhibition, $\mathrm{C}=$ Radial growth in control $(\mathrm{mm}), \mathrm{T}=$ Radial growth in treatment $(\mathrm{mm})$

\section{Results and Discussion}

A total 66 fungal (26 from root, 15 from stem and 26 from leaf) endophytic isolates were obtained from different parts of healthy tomato samples, which were collected from 30 locations in three districts of northern Karnataka. A total of 66 fungal endophytes were evaluated against $S$. rolfsii, $R$. solani and $F$. solani by dual culture technique to test antifungal activity of the endophytes.

A total of 26 fungal root endophytes were evaluated against three pathogens by dual culture technique and results are presented in Table 1. The maximum mycelial inhibition against $S$. rolfsii was observed by the root endophytic isolates RFBBA-23 (70.59\%) and RFHKM-9 (69.02\%), which were on par with each other. The isolate RFHAR-6 showed the least mycelial inhibition $(29.80 \%)$. Isolate RFHKM-9 showed the maximum mycelial inhibition of 60.39 per cent against $R$. solani which was followed by RFBBA- $23(54.51 \%)$ and RFDHE-10 (51.76). The isolates RFHAR5, RFDHE-11 and RFBSA-25 did not show any inhibition against $R$. solani. Isolates RFHHO-7 and RFBBA-23 showed maximum mycelial inhibition of 79.22 and 77.65 per cent, respectively against $F$. solani and these were on par with each other. The isolate
RFBHU-26 showed the least inhibition (41.96) as compared to other isolates. Overall the isolates RFHHO- 7, RFHKM-9, RFDHE10 and RFBBA-23 were effective against all the three pathogens and isolates were less effective against $R$. solani as compared to other two pathogens.

A total of 15 fungal stem endophytes were evaluated against three pathogens by dual culture technique and results are presented in Table 2. The maximum mycelial inhibition of S. rolfsii was showed by the stem endophytic isolate SFDOF-11 (69.02\%) followed by SFDDE-12 (64.31\%) and isolate SFBHU-15 showed the least mycelial inhibition (23.14 $\%)$. Isolate SFDDE-12 showed the maximum mycelial inhibition of 61.96 per cent against $R$. solani followed by SFDDE-12 (54.90 \%) and isolates SFDUN-13 and SFBBE-14 did not show any inhibition against $R$. solani. Isolate SFDOF-11 showed the maximum mycelial inhibition of 69.41 per cent against F. solani followed by SFDDE-12 (65.10\%) and isolate SFBHU-15 (38.82\%) showed the least mycelial inhibition as compared to other isolates. Overall the isolates SFDOF-11 and SFDDE-12 were effective against all the three pathogens.

A total of 26 fungal leaf endophytes were evaluated against three pathogens by dual culture technique and results are presented in Table 3. The maximum mycelial inhibition of $S$. rolfsii was shown by the leaf endophytic isolate LFDLA-9 $(65.10 \%)$ followed by LFDHO-3 (64.31\%) and isolate LFHHO-7 showed the least mycelial inhibition (18.04 $\%)$. The maximum mycelial inhibition of $R$. solani was shown by the isolates LFDHO-3 (52.22 \%), LFDLA-9 (51.48), LFHCH-13 (50.37), LFHCH-12 (49.63\%) and LFHMU$16(49.63 \%)$, these were on par with each other and isolate LFHHO-7 did not show any inhibition of $R$. solani. 
Table.1 In vitro evaluation of fungal root endophytes against Sclerotium rolfsii, Rhizoctonia solani and Fusarium solani by dual culture method

\begin{tabular}{|c|c|c|c|}
\hline \multirow[t]{2}{*}{ Isolate } & \multicolumn{3}{|c|}{ Per cent inhibition over control } \\
\hline & S. rolfsii & R. solani & F. solani \\
\hline RFDGP-1 & $\begin{array}{c}41.96 \\
(40.36)^{*}\end{array}$ & $\begin{array}{c}38.04 \\
(38.06)\end{array}$ & $\begin{array}{c}51.37 \\
(45.77)\end{array}$ \\
\hline RFDHO-2 & $\begin{array}{c}40.78 \\
(39.67)\end{array}$ & $\begin{array}{c}20.39 \\
(26.83)\end{array}$ & $\begin{array}{c}46.67 \\
(43.07)\end{array}$ \\
\hline RFDHO-3 & $\begin{array}{c}34.12 \\
(35.73)\end{array}$ & $\begin{array}{c}17.25 \\
(24.53)\end{array}$ & $\begin{array}{c}49.80 \\
(44.87)\end{array}$ \\
\hline RFHNI-4 & $\begin{array}{c}32.94 \\
(35.01)\end{array}$ & $\begin{array}{c}20.78 \\
(27.11)\end{array}$ & $\begin{array}{c}46.27 \\
(42.85)\end{array}$ \\
\hline RFHAR-5 & $\begin{array}{c}36.08 \\
(36.90)\end{array}$ & $\begin{array}{c}0.00 \\
(0.00)\end{array}$ & $\begin{array}{c}46.67 \\
(43.07)\end{array}$ \\
\hline RFHKA-6 & $\begin{array}{c}29.80 \\
(33.07)\end{array}$ & $\begin{array}{c}23.92 \\
(29.27)\end{array}$ & $\begin{array}{c}50.98 \\
(45.54)\end{array}$ \\
\hline RFHHO-7 & $\begin{array}{c}58.04 \\
(49.61)\end{array}$ & $\begin{array}{c}50.59 \\
(45.77)\end{array}$ & $\begin{array}{c}79.22 \\
(62.85)\end{array}$ \\
\hline RFDLA-8 & $\begin{array}{c}38.43 \\
(38.29)\end{array}$ & $\begin{array}{c}31.37 \\
(34.05)\end{array}$ & $\begin{array}{c}60.78 \\
(51.21)\end{array}$ \\
\hline RFHKM-9 & $\begin{array}{c}69.02 \\
(56.17)\end{array}$ & $\begin{array}{c}60.39 \\
(50.98)\end{array}$ & $\begin{array}{c}76.08 \\
(60.69)\end{array}$ \\
\hline RFDHE-10 & $\begin{array}{c}65.10 \\
(53.77)\end{array}$ & $\begin{array}{c}51.76 \\
(45.99)\end{array}$ & $\begin{array}{c}76.08 \\
(60.69)\end{array}$ \\
\hline RFDHE-11 & $\begin{array}{c}38.04 \\
(38.06)\end{array}$ & $\begin{array}{c}0.00 \\
(0.00)\end{array}$ & $\begin{array}{c}47.45 \\
(43.52)\end{array}$ \\
\hline RFHBA-12 & $\begin{array}{c}40.39 \\
(39.44)\end{array}$ & $\begin{array}{c}35.69 \\
(36.67)\end{array}$ & $\begin{array}{c}47.45 \\
(43.52)\end{array}$ \\
\hline RFDOF-13 & $\begin{array}{c}36.08 \\
(36.90)\end{array}$ & $\begin{array}{c}27.06 \\
(31.33)\end{array}$ & $\begin{array}{c}52.55 \\
(46.44)\end{array}$ \\
\hline RFDOF-14 & $\begin{array}{c}33.33 \\
(35.25)\end{array}$ & $\begin{array}{c}33.33 \\
(35.25)\end{array}$ & $\begin{array}{c}51.37 \\
(45.77)\end{array}$ \\
\hline RFDDE-45 & $\begin{array}{c}39.61 \\
(38.98)\end{array}$ & $\begin{array}{c}25.49 \\
(30.31)\end{array}$ & $\begin{array}{c}54.51 \\
(47.57)\end{array}$ \\
\hline RFDCH-16 & $\begin{array}{c}30.98 \\
(33.80)\end{array}$ & $\begin{array}{c}30.98 \\
(33.81)\end{array}$ & $\begin{array}{c}56.86 \\
(48.92)\end{array}$ \\
\hline RFDNU-17 & $\begin{array}{c}36.47 \\
(37.13)\end{array}$ & $\begin{array}{c}32.55 \\
(34.77)\end{array}$ & $\begin{array}{c}46.27 \\
(42.85)\end{array}$ \\
\hline RFDVA-18 & $\begin{array}{c}40.39 \\
(39.44)\end{array}$ & $\begin{array}{c}29.80 \\
(33.07)\end{array}$ & $\begin{array}{c}47.45 \\
(43.52)\end{array}$ \\
\hline RFBBE-19 & $\begin{array}{c}35.69 \\
(36.66)\end{array}$ & $\begin{array}{c}47.06 \\
(43.30)\end{array}$ & $\begin{array}{c}58.04 \\
(49.61)\end{array}$ \\
\hline RFBBE-20 & $\begin{array}{c}38.43 \\
(38.29)\end{array}$ & $\begin{array}{c}45.88 \\
(42.62)\end{array}$ & $\begin{array}{c}46.27 \\
(42.85)\end{array}$ \\
\hline RFDUN-21 & $\begin{array}{c}34.90 \\
(36.20)\end{array}$ & $\begin{array}{c}37.25 \\
(37.60)\end{array}$ & $\begin{array}{c}52.16 \\
(46.22)\end{array}$ \\
\hline RFDUN-22 & $\begin{array}{c}47.06 \\
(43.30)\end{array}$ & $\begin{array}{c}50.20 \\
(45.09)\end{array}$ & $\begin{array}{c}62.75 \\
(52.36)\end{array}$ \\
\hline RFBBA-23 & $\begin{array}{c}70.59 \\
(57.13)\end{array}$ & $\begin{array}{c}54.51 \\
(47.57)\end{array}$ & $\begin{array}{c}77.65 \\
(61.76)\end{array}$ \\
\hline RFBBA-24 & $\begin{array}{c}40.39 \\
(39.44)\end{array}$ & $\begin{array}{c}28.63 \\
(32.33)\end{array}$ & $\begin{array}{c}45.49 \\
(42.40)\end{array}$ \\
\hline RFBSA-25 & $\begin{array}{c}36.47 \\
(37.14)\end{array}$ & $\begin{array}{c}0.00 \\
(0.00)\end{array}$ & $\begin{array}{c}63.14 \\
(52.60)\end{array}$ \\
\hline RFBHU-26 & $\begin{array}{c}30.20 \\
(33.31)\end{array}$ & $\begin{array}{c}13.33 \\
(21.41)\end{array}$ & $\begin{array}{c}41.96 \\
(40.36)\end{array}$ \\
\hline S.Em. \pm & 0.37 & 0.47 & 0.33 \\
\hline C.D. at $1 \%$ & 1.40 & 1.76 & 1.24 \\
\hline
\end{tabular}

* Arcsine transformed values 
Table.2 In vitro evaluation of fungal stem endophytes against Sclerotium rolfsii, Rhizoctonia solani and Fusarium solani by dual culture method

\begin{tabular}{|c|c|c|c|}
\hline \multirow[t]{2}{*}{ Isolate } & \multicolumn{3}{|c|}{ Per cent inhibition over control } \\
\hline & S. rolfsii & R. solani & F. solani \\
\hline SFDHO-1 & $\begin{array}{c}41.18 \\
(39.90)^{*}\end{array}$ & $\begin{array}{c}41.96 \\
(40.36)\end{array}$ & $\begin{array}{c}56.86 \\
(48.92)\end{array}$ \\
\hline SFHNI-2 & $\begin{array}{c}35.69 \\
(36.67)\end{array}$ & $\begin{array}{c}38.43 \\
(38.30)\end{array}$ & $\begin{array}{c}50.20 \\
(45.09)\end{array}$ \\
\hline SFHKA-3 & $\begin{array}{c}38.43 \\
(38.30)\end{array}$ & $\begin{array}{c}36.47 \\
(37.14)\end{array}$ & $\begin{array}{c}53.33 \\
(46.89)\end{array}$ \\
\hline SFDLA-4 & $\begin{array}{c}45.88 \\
(42.62)\end{array}$ & $\begin{array}{c}29.02 \\
(32.58)\end{array}$ & $\begin{array}{c}46.27 \\
(42.85)\end{array}$ \\
\hline SFDHE-5 & $\begin{array}{c}36.86 \\
(37.37)\end{array}$ & $\begin{array}{c}30.98 \\
(33.81)\end{array}$ & $\begin{array}{c}47.45 \\
(43.52)\end{array}$ \\
\hline SFDHE-6 & $\begin{array}{c}26.67 \\
(31.08)\end{array}$ & $\begin{array}{c}45.88 \\
(42.62)\end{array}$ & $\begin{array}{c}46.27 \\
(42.85)\end{array}$ \\
\hline SFHKM-7 & $\begin{array}{c}32.94 \\
(35.01)\end{array}$ & $\begin{array}{c}43.92 \\
(41.49)\end{array}$ & $\begin{array}{c}47.84 \\
(43.75)\end{array}$ \\
\hline SFHRA-8 & $\begin{array}{c}29.80 \\
(33.07)\end{array}$ & $\begin{array}{c}46.27 \\
(42.85)\end{array}$ & $\begin{array}{c}52.55 \\
(46.44)\end{array}$ \\
\hline SFHBK-9 & $\begin{array}{c}29.02 \\
(32.58)\end{array}$ & $\begin{array}{c}24.71 \\
(29.79)\end{array}$ & $\begin{array}{c}40.00 \\
(39.22)\end{array}$ \\
\hline SFHMU-10 & $\begin{array}{c}35.69 \\
(36.67)\end{array}$ & $\begin{array}{c}41.18 \\
(39.90)\end{array}$ & $\begin{array}{c}46.67 \\
(43.07)\end{array}$ \\
\hline SFDOF-11 & $\begin{array}{c}69.02 \\
(56.16)\end{array}$ & $\begin{array}{c}54.90 \\
(47.79)\end{array}$ & $\begin{array}{c}69.41 \\
(56.40)\end{array}$ \\
\hline SFDDE-12 & $\begin{array}{c}64.31 \\
(53.30)\end{array}$ & $\begin{array}{c}61.96 \\
(51.90)\end{array}$ & $\begin{array}{c}65.10 \\
(53.77)\end{array}$ \\
\hline SFDUN-13 & $\begin{array}{c}49.02 \\
(44.42)\end{array}$ & $\begin{array}{c}0.00 \\
(0.00)\end{array}$ & $\begin{array}{c}44.31 \\
(41.72)\end{array}$ \\
\hline SFBBE-14 & $\begin{array}{c}32.16 \\
(34.53)\end{array}$ & $\begin{array}{c}0.00 \\
(0.00)\end{array}$ & $\begin{array}{c}46.67 \\
(43.07)\end{array}$ \\
\hline SFBHU-15 & $\begin{array}{c}23.14 \\
(28.74)\end{array}$ & $\begin{array}{c}8.24 \\
(16.67)\end{array}$ & $\begin{array}{c}38.82 \\
(38.53)\end{array}$ \\
\hline S.Em. \pm & 0.28 & 0.20 & 0.29 \\
\hline C.D. at $1 \%$ & 1.09 & 0.77 & 1.13 \\
\hline
\end{tabular}

* Arcsine transformed values 
Table.3 In vitro evaluation of fungal leaf endophytes against Sclerotium rolfsii, Rhizoctonia solani and Fusarium solani by dual culture method

\begin{tabular}{|c|c|c|c|}
\hline \multirow[t]{2}{*}{ Isolate } & \multicolumn{3}{|c|}{ Per cent inhibition over control } \\
\hline & S. rolfsii & R. solani & F. solani \\
\hline LFDGP-1 & $\begin{array}{c}44.71 \\
(41.94)^{*}\end{array}$ & $\begin{array}{c}37.41 \\
(37.69)\end{array}$ & $\begin{array}{c}44.31 \\
(41.72)\end{array}$ \\
\hline LFDGP-2 & $\begin{array}{c}39.61 \\
(38.99)\end{array}$ & $\begin{array}{c}33.70 \\
(35.47)\end{array}$ & $\begin{array}{c}40.78 \\
(39.67)\end{array}$ \\
\hline LFDHO-3 & $\begin{array}{c}64.31 \\
(53.30)\end{array}$ & $\begin{array}{c}52.22 \\
(46.26)\end{array}$ & $\begin{array}{c}59.22 \\
(50.29)\end{array}$ \\
\hline LFHNI-4 & $\begin{array}{c}41.18 \\
(39.90)\end{array}$ & $\begin{array}{c}9.63 \\
(18.07)\end{array}$ & $\begin{array}{c}50.59 \\
(45.32)\end{array}$ \\
\hline LFHAR-5 & $\begin{array}{c}40.78 \\
(39.67)\end{array}$ & $\begin{array}{c}28.15 \\
(32.03)\end{array}$ & $\begin{array}{c}47.84 \\
(43.75)\end{array}$ \\
\hline LFHHO-6 & $\begin{array}{c}43.14 \\
(41.04)\end{array}$ & $\begin{array}{c}40.74 \\
(39.65)\end{array}$ & $\begin{array}{c}47.84 \\
(43.75)\end{array}$ \\
\hline LFHHO-7 & $\begin{array}{c}18.04 \\
(25.12)\end{array}$ & $\begin{array}{c}0.00 \\
(0.00)\end{array}$ & $\begin{array}{c}38.04 \\
(38.06)\end{array}$ \\
\hline LFDNA-8 & $\begin{array}{c}45.88 \\
(42.62)\end{array}$ & $\begin{array}{c}41.85 \\
(40.29)\end{array}$ & $\begin{array}{c}42.75 \\
(40.81)\end{array}$ \\
\hline LFDLA -9 & $\begin{array}{c}65.10 \\
(53.77)\end{array}$ & $\begin{array}{c}51.48 \\
(45.83)\end{array}$ & $\begin{array}{c}67.84 \\
(55.43)\end{array}$ \\
\hline LFHKM-10 & $\begin{array}{c}27.84 \\
(31.84)\end{array}$ & $\begin{array}{c}36.67 \\
(37.25)\end{array}$ & $\begin{array}{c}45.88 \\
(42.62)\end{array}$ \\
\hline LFHKM-11 & $\begin{array}{c}34.51 \\
(35.96)\end{array}$ & $\begin{array}{c}44.07 \\
(41.58)\end{array}$ & $\begin{array}{c}46.67 \\
(43.07)\end{array}$ \\
\hline LFHCH-12 & $\begin{array}{c}27.84 \\
(31.84)\end{array}$ & $\begin{array}{c}49.63 \\
(44.77)\end{array}$ & $\begin{array}{c}51.76 \\
(45.99)\end{array}$ \\
\hline LFHCH-13 & $\begin{array}{c}38.04 \\
(38.06)\end{array}$ & $\begin{array}{c}50.37 \\
(45.19)\end{array}$ & $\begin{array}{c}47.84 \\
(43.75)\end{array}$ \\
\hline LFHBA-14 & $\begin{array}{c}36.86 \\
(37.37)\end{array}$ & $\begin{array}{c}40.74 \\
(39.65)\end{array}$ & $\begin{array}{c}43.14 \\
(41.04)\end{array}$ \\
\hline LFHMU -15 & $\begin{array}{c}35.69 \\
(36.67)\end{array}$ & $\begin{array}{c}42.59 \\
(40.72)\end{array}$ & $\begin{array}{c}49.80 \\
(44.87)\end{array}$ \\
\hline LFHMU-16 & $\begin{array}{c}43.92 \\
(41.49)\end{array}$ & $\begin{array}{c}49.63 \\
(44.77)\end{array}$ & $\begin{array}{c}50.20 \\
(45.09)\end{array}$ \\
\hline LFDOF-17 & $\begin{array}{c}27.84 \\
(31.84)\end{array}$ & $\begin{array}{c}44.07 \\
(41.58)\end{array}$ & $\begin{array}{c}51.37 \\
(45.77)\end{array}$ \\
\hline LFDDE-18 & $\begin{array}{c}21.18 \\
(27.39)\end{array}$ & $\begin{array}{c}45.19 \\
(42.22)\end{array}$ & $\begin{array}{c}55.29 \\
(48.02)\end{array}$ \\
\hline LFDKA-19 & $\begin{array}{c}26.27 \\
(30.82)\end{array}$ & $\begin{array}{c}34.07 \\
(35.70)\end{array}$ & $\begin{array}{c}49.41 \\
(44.64)\end{array}$ \\
\hline LFDKA-20 & $\begin{array}{c}48.24 \\
(43.97)\end{array}$ & $\begin{array}{c}41.85 \\
(40.29)\end{array}$ & $\begin{array}{c}47.84 \\
(43.75)\end{array}$ \\
\hline LFDNU-21 & $\begin{array}{c}29.80 \\
(33.07)\end{array}$ & $\begin{array}{c}41.85 \\
(40.29)\end{array}$ & $\begin{array}{c}49.02 \\
(44.42)\end{array}$ \\
\hline LFDUN-22 & $\begin{array}{c}32.55 \\
(34.77)\end{array}$ & $\begin{array}{c}37.78 \\
(37.91)\end{array}$ & $\begin{array}{c}45.10 \\
(42.17)\end{array}$ \\
\hline LFBHA-23 & $\begin{array}{c}43.14 \\
(41.04)\end{array}$ & $\begin{array}{c}43.33 \\
(41.15)\end{array}$ & $\begin{array}{c}47.45 \\
(43.52)\end{array}$ \\
\hline LFBHU-24 & $\begin{array}{c}40.78 \\
(39.67)\end{array}$ & $\begin{array}{c}38.15 \\
(38.13)\end{array}$ & $\begin{array}{c}50.20 \\
(45.09)\end{array}$ \\
\hline LFBSA-25 & $\begin{array}{c}46.27 \\
(42.85)\end{array}$ & $\begin{array}{c}45.56 \\
(42.43)\end{array}$ & $\begin{array}{c}51.37 \\
(45.77)\end{array}$ \\
\hline S.Em. \pm & 0.37 & 0.30 & 0.40 \\
\hline C.D. at $1 \%$ & 1.39 & 1.12 & 1.51 \\
\hline
\end{tabular}

* Arcsine transformed values 
Isolate LFDLA-9 showed the maximum mycelial inhibition of 67.84 per cent against F. solani followed by LFDHO-3 (59.22 \%) and the least mycelial inhibition was observed by LFDGP-2 (40.78\%). Overall the isolates LFDHO-3 and LFDLA-9 were effective against all the three pathogens. Though isolate LFDKA-20 showed less inhibition of all three pathogens in comparison with other isolates, it showed the clear inhibition zone against all the three pathogens. The findings of the present study are in agreement with Narayan et al., (2012) and Ngatia et al., (2015) who evaluated fungal endophytes against chilli pathogens (Fusarium oxysporum, Phytophthora capsici and Colletotrichum acutatum) and tomato pathogen (P. infestans causes late blight), respectively by employing dual culture method. Mousa and Raizada (2013) reviewed diverse classes of secondary metabolites, focusing on antimicrobial compounds, synthesized by endophytes including terpenoids, alkaloids, phenylpropanoids, aliphatic compounds, polyketides and peptides from the interdisciplinary perspectives of biochemistry, genetics, fungal biology, host plant biology, human and plant pathology. Many endophytes produce secondary metabolites such as auxin, gibberellin etc. that help in growth and development of the host plant. Some of these compounds are antibiotics having antifungal, antibacterial and insecticidal properties, which may inhibit the growth of plant pathogens. The extent of inhibition of three pathogens by fungal endophytes in dual culture method ranged from 0.00 to 79.22 percent. From the results of present study it is concluded that the possible role of growth inhibition by the endophytes was attributed to the production of diffusible and volatile metabolites, lytic enzymes and by competition with pathogens.

It is concluded that among the 66 fungal isolates, irrespective of isolates from root, stem and leaf, some (RFHHO-7, RFHKM-9, RFDHE-10, RFBBA-23, SFDOF-11, SFDDE-12, LFDHO-3 and LFDLA-9) have shown maximum mycelial inhibition of three pathogens. Isolates RFBBE-19 and RFDUN22 were fast growing isolates and were more effective against $F$. solani and $R$. solani as compared to $S$. rolfsii. Isolate LFDKA-20 though showed the minimum inhibition against all pathogens as compared to other effective isolates but it also showed the clear inhibition zone which may be due to the production of antimicrobial compounds from the endophyte. From the results of present study it is concluded that the possible role of growth inhibition by the endophytes was attributed to the production of diffusible and volatile metabolites, lytic enzymes and by competition with pathogens.

\section{References}

Anonymous, 2017, Ministry of Agriculture and Farmers Welfare, Govt. of India.

Deepa, J. and Sally, K., 2015, Antagonistic activity of endophytic microorganisms against bacterial wilt disease of tomato. Inter. J. Current Advanced Res., 4 (10): 399-404.

Dias, J. S., 2012, Nutritional quality and health benefits of vegetables: A review. Food Nutrition Sci., 3: 13541374.

Kumar, N. and Sharma, S., 2015, Fusarial wilt of Solanum lycopersicum L. (tomato) at Panchgaon. Int. J. Curr. Microbiol. App. Sci., 4(11): 253-260.

Mandal, A. K., Praveen, K. M., Subrata, D. and Arup, C., 2017, Effective management of major tomato diseases in the gangetic plains of eastern India through integrated approach. Agri. Res. Tech., 10(5): DOI: 10.19080.

Mousa, W. and Raizada, N., 2012, The diversity of anti-microbial secondary metabolites produced by fungal 
endophytes: An interdisciplinary perspective. Frontiers Microbiol., 65(4): 18-36.

Muriungi, S. J., Mutitu, E. W. and Muthomi, J. W., 2014, Efficacy of cultural methods in the control of Rhizoctonia solani strains causing tomato damping off in Kenya. African J. Food Agri. Nutri. Develop., 14(2): 8776-8790.

Narayan, C. P., Jian, X. D., Hyun, K. S., Young, P. C. and Seung, H., 2012, Distribution and antifungal activity of endophytic fungi in different growth stages of chilli pepper (Capsicum aпnиum L.) in Korea. Pl. Pathol. J., 28 (1): 10-19.

Ngatia, G., El-Hasan, A. and Vogele, R., 2015, Antagonistic activity of fungal root endophytes from solanaceous plants against potato late blight (Phytophthora infestans). In proceedings of the $18^{\text {th }}$ conference of
International Plant Protection Congress (IPPC), Berlin, 24-27 August 2015.

Pawle, G. and Singh, S. K., 2014, Antimicrobial, antioxidant activity and phytochemical analysis of an endophytic species of Nigrospora isolated from living fossil Ginkgo biloba. Curr. Res. Environ. App. Myco., 4(1): 1-9.

Schulz, B., Wanke, U. and Draeger, S., 1993, Endophytes from herbaceous and shrubs: effectiveness of surface sterilization methods. Mycol. Res., 97:1447-50.

Steven, T. K., 2003, Commercial greenhouse vegetable handbook. ANR publications, California, pp, 1-13.

Vincent, J. M., 1947, Distortion of fungal hyphae in the presence of certain inhibitors. Nature, 150: 850-857.

\section{How to cite this article:}

Basamma Hadimani and Naik, S.T. 2018. Screening of Fungal Endopohytes against Soil-borne Fungal Pathogens in Tomato. Int.J.Curr.Microbiol.App.Sci. 7(07): 3534-3541.

doi: https://doi.org/10.20546/ijcmas.2018.707.410 